\title{
Ciclo de Mejora en el Aula en los estudios de Gestión de Enfermería: Evaluación orientada hacia el aprendizaje
}

\section{Classroom Improvement Cycle in Nursing Management studies: Evaluation oriented towards learning}

MARÍA DOLORES GUERRA-MARTíN

ORCID: https://orcid.org/0000-0002-1409-1287

Universidad de Sevilla.

Departamento de Enfermería

guema@us.es

Fecha de recepción:

Fecha de aceptación:

DOI: http://dx.doi.org/10.12795/9788447221912.094

Pp.: 2116-2143 


\section{Resumen}

El Ciclo de Mejora en el Aula se ha realizado en la asignatura de Ética y Gestión de Enfermería, de 3er curso del Grado en Enfermería, de la Universidad de Sevilla. Este se ha llevado a cabo en un grupo grande con 61 estudiantes, y en un grupo pequeño con 13 estudiantes. La duración del mismo ha sido de 34 horas, de las cuales 22 se han impartido en grupo grande y 12 en grupo pequeño, desde el 26/09/19 hasta el 15/11/19. La parte en la cual se ha llevado a cabo esta experiencia docente, ha sido en la de Gestión de Enfermería. En grupo grande la docencia ha sido interactiva, incorporando el Trabajo en Equipo Colaborativo entre los estudiantes y Búsquedas de Información Autónomas, y en el grupo pequeño se ha combinado esto último, junto con el Aula Invertida y el Aprendizaje Basado en Problemas.

Palabras clave: Gestión de Enfermería, Grado en Enfermería; Docencia Universitaria, Experimentación Docente Universitaria, Evaluación orientada hacia el aprendizaje.

\section{Abstract}

The Cycle of improvement in the classroom has been carried out in the subject of Ethics and Nursing Management, 3rd year of the Degree in Nursing, of the University of Seville. This has been carried out in a large group with 61 students, and in a small group with 13 students. Its duration has been 34 hours, of which 22 have been taught in large groups, and 12 in small groups, from 09/26/19 to 11/15/19. The part in which this teaching experience has been carried out has been in Nursing Management. In large group the teaching has been interactive, incorporating the Collaborative Teamwork among students, and Autonomous Information Searches, and in a small group the latter has been combined, together with the Inverted Classroom and Problem Based Learning.

Keywords: Nursing Management, Degree in Nursing; University Teaching, University Teaching Experimentation, Evaluation oriented towards learning. 


\section{Descripción del contexto}

En la asignatura de Ética y Gestión de Enfermería, se han llevado a cabo tres CIMA encadenados, dicha asignatura es de 3er curso del Grado en Enfermería, de la Facultad de Enfermería, Fisioterapia y Podología de la Universidad de Sevilla (US). Esta asignatura es obligatoria, se imparte en el primer cuatrimestre, y tiene seis créditos ECTS, de los cuáles, cuatro se imparten en grupo grande (por las mañanas) y dos en pequeños grupos (por las tardes). Esta facultad tiene tres Unidades Docentes (UD) cercanas a los hospitales de referencia; en la UD Macarena donde hay establecidos dos grupos grandes, en la de Valme uno y en la de Rocío otro. Respecto a los grupos pequeños (subgrupos) hay 16 , seis en UD Macarena, cuatro en la de Valme y seis en la de Rocío.

Se han realizado dos CIMA en un grupo grande de la UD Macarena, con 61 estudiantes y en un grupo pequeño de talleres con 13 estudiantes. La duración del CIMA ha sido de 34 horas, de las cuales, 22 se han impartido en grupo grande, y 12 en grupo pequeño, desde el 26/09/19 hasta el $15 / 11 / 19$.

La docencia en grupo grande se ha organizado en dos bloques, uno sobre sistemas sanitarios-CIMA 1 (cuatro temas, desde lo más general, en cuanto al inicio y desarrollo de los sistemas de salud, hasta lo más específico, en relación al Sistema Sanitario Público Andaluz) y otro sobre el proceso administrativo- CIMA 2 (cuatro temas, desarrollando las etapas de dicho proceso), y en la de grupo pequeño- CIMA 3, se han trabajado distintas competencias (una relacionada con el trabajo en equipo, dos con los sistemas sanitarios y tres con el proceso administrativo).

Esta asignatura la llevo impartiendo desde la implantación del Grado en Enfermería en la US (curso 2009/2010), $y$ he realizado anteriormente tres CIMA. En los curso 2014/2015 y 2017/2018 (Guerra-Martín, 2015 y Guerra-Martín, 2017) las experiencias educativas fueron con grupos 
pequeños y no fue hasta que conseguí conjugar mi modelo metodológico posible con el ideal, cuando me vi preparada para experimentarlo en grupo grande. En el curso 2018/2019 (Guerra-Martín, 2018) llevé a cabo un CIMA en un grupo grande, y en el curso actual 2019/2020, he integrado en los ciclos de mejora, tanto la docencia en grupo grande como en grupo pequeño.

\section{Conexión con el diseño previo}

En el curso 2018/2019 el planteamiento de la clase, fue el siguiente: Al inicio de la misma hacía una sintesis de la materia a tratar y/o de lo impartido en la clase anterior, después los estudiantes trabajaban en equipo, de una manera colaborativa, realizando lecturas del material docente expuesto en la plataforma virtual, posteriormente, estos planteaban sus dudas, que intentaban resolverlas mediante búsquedas autónomas de información. Si había alguna cuestión en la cual hubiera discrepancia, se ponían en contacto conmigo para orientarles al respecto, finalizado este proceso, los estudiantes elaboraban preguntas tipo test, con tres opciones de respuesta, de las cuáles, una era verdadera. Estas las iban introduciendo en la plataforma Kahoot, para después ponerlas en clase en grupo grande y que los estudiantes las contestaran, de esta manera, valoraban los conocimientos que iban adquiriendo, lo cual les servía como mecanismo de mejora del proceso de aprendizaje, ya que detectaban errores y los iban corrigiendo sobre la marcha. En la última clase dejaba un tiempo para realizar una sintesis y conclusiones del tema, y para recordarles el tema siguiente del que debían hacer lecturas relacionadas con el mismo.

Durante este curso 2019/2020, he continuado con el mismo planteamiento de las clases llevado a cabo en el curso anterior, aunque en este he realizado las mejoras en base a las experiencias adquiridas, por ejemplo, en el curso anterior, se realizaron bastantes Kahoot, lo cual fue un trabajo excesivo, siendo esta opinión compartida por 
muchos estudiantes (algunos comentaron que ya estaban hartos del mismo), por lo que, este curso se han realizado dos Kahoot, uno por cada dos temas, aunque, con mayor número de preguntas y con una mayor profundización. Además, los estudiantes me plantearon que debía hacer una mayor presentación al inicio de cada tema, antes del trabajo en equipo, lo que he tenido en cuenta en Ios CIMA 1 y 2. En cuanto al CIMA 3 en grupo pequeño se ha actualizado la guía de los talleres con los objetivos, contenidos, procedimientos y evaluación establecidos para cada uno.

Respecto a la evaluación y calificación, se han planteado dos modalidades, una para grupo grande (examen tipo test, que se ha trabajado mediante Kahoot) y otra para grupo pequeño (mediante evaluación continua). El examen tipo test, aunque puede parecer discordante con la metodología de trabajo seguida en los grupos grandes, se fundamenta en que los estudiantes, una vez que finalizan la carrera, la mayoría se presentan a la convocatoria de Enfermero Interno Residente (EIR), que es un sistema por el cual los enfermeros españoles pueden acceder a las plazas de formación especializada (Salud Mental, Obstétrico-ginecológica, Familiar y Comunitaria, entre otras), conteniendo más de 200 preguntas tipo test, siendo muchas de ellas sobre temas de gestión de Enfermería. En este sentido, los estudiantes de cursos anteriores han ido manifestando su satisfacción con este tipo de preguntas, ya que están próximos a finalizar sus estudios, por lo que, las valoran como método de entrenamiento para poder aprobar el examen EIR y sacar una plaza de Enfermero/a Especialista. En este sentido, decir que la evaluación está orientando hacia el aprendizaje, ya que los estudiantes conocen a priori lo que tienen que conseguir (Padilla-Carmona, Gil-Flores, Rodríguez-Santero, Torres-Gordillo y Clares-López, 2010). Además, estos estudiantes participan en su proceso de enseñanza-aprendizaje, siendo las búsquedas de información autónomas, fundamentales para el aprendizaje a lo largo de la vida. Referir que con el examen los estudiantes pueden conseguir una calificación de ocho puntos, sobre diez. 
En cuanto al grupo pequeño, se pueden conseguir dos puntos, sobre diez, mediante una evaluación continua (feedforward) y la entrega de un informe del trabajo en equipo. Se han tenido en cuenta para la evaluación de los talleres dos apartados con los siguientes criterios: Presencialidad: Puntualidad, participación, actitud proactiva y respeto de las normas del equipo. Informes: Presentación, puntualidad en la entrega y calidad de la contribución (incluyendo la bibliografia).

\section{Modelo metodológico}

El modelo metodológico a seguir en grupo grande, ha sido el mismo que llevé a cabo en el curso anterior, con la salvedad de que he utilizado algo más de tiempo al inicio de la clase para la presentación del tema y problemas, y menos uso de la herramienta Kahoot, como he referido anteriormente. En la figura 1, se representa dicho modelo.

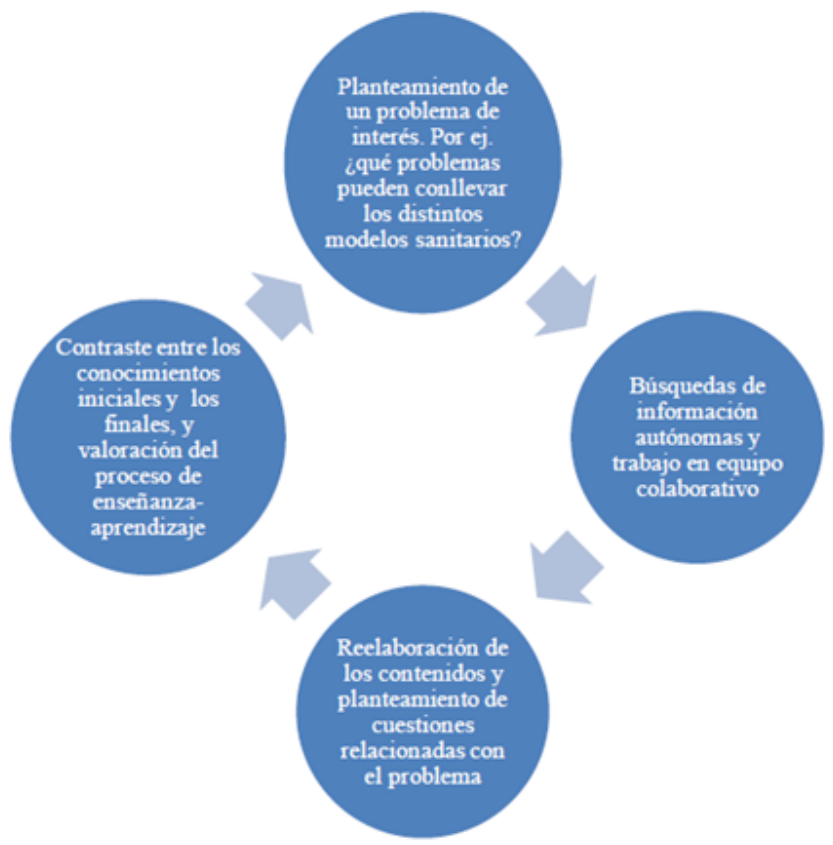

Figura 1. Representación de mi modelo metodológico en grupo grande. Basado en De Alba y Porlán, 2017.

Jornadas de Formación e Innovación Docente del Profesorado | № 2 (2019) Esta obra se distribuye con la licencia Creative Commons Reconocimiento-NoComercial-SinObraDerivada 
El modelo metodológico seguido en grupo pequeño (Figura 2), está basado en el Aula Invertida (Fernández-Gámez y Guerra-Martín, 2016; Tourón y Santiago, 2015), y en el Aprendizaje Basado en Problemas (Gil-Galván, 2018; Guerra, 2009), ya que las búsquedas de información las han realizado los estudiantes, mayormente, fuera del aula disponiendo de una semana para las mismas, y en el aula (talleres), han trabajado en equipo, poniendo de manifiesto las dudas, los problemas a la hora de realizar las búsquedas de información, las discrepancias, entre otras, y volviendo a hacer nuevas búsquedas en el aula, para posteriormente, realizar una puesta en común y debate; finalizando el taller, con una sintesis y conclusiones por parte de la profesora. Como se ha comentado antes, se ha llevado a cabo una evaluación continua con retroalimentación y proalimentación (feedforward) (Guerra-Martín, Fernández-Rodríguez, Lima-Serrano y Rodríguez-Borrego, 2012), siendo esta una de las condiciones para que la evaluación se oriente hacia el aprendizaje, a la vez que, se ha implicado a los estudiantes de manera activa para promover el aprendizaje a lo largo de la vida (Padilla-Carmona, Gil-Flores, Rodríguez-Santero, Torres-Gordillo y Clares-López, 2010).

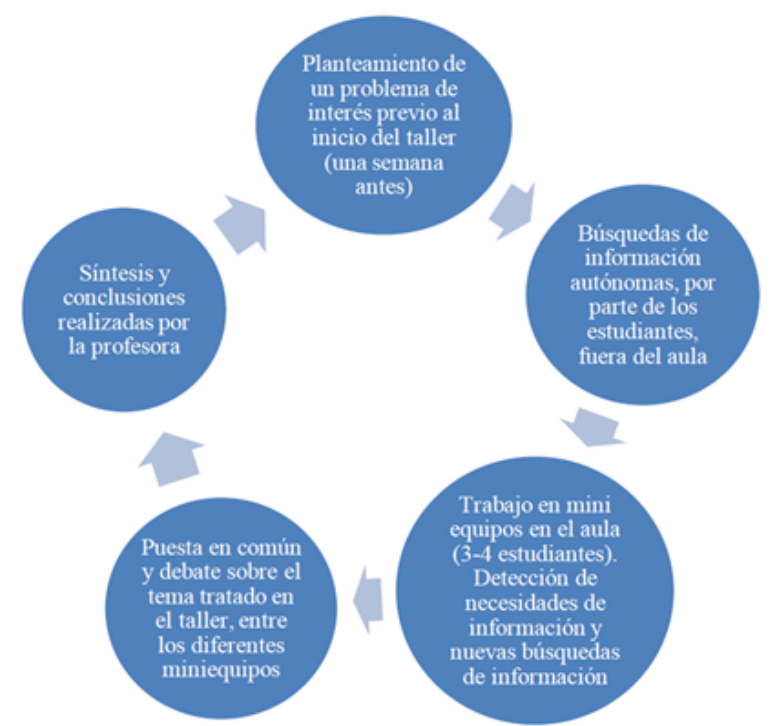

Figura 2. Representación de mi modelo metodológico, en pequeño grupo.

Jornadas de Formación e Innovación Docente del Profesorado | № 2 (2019) Esta obra se distribuye con la licencia Creative Commons Reconocimiento-NoComercial-SinObraDerivada 


\section{Mapas de contenido}

Como se ha comentado anteriormente, se han llevado a cabo tres CIMA encadenados, el primero y el segundo en el grupo grande y el tercero en el grupo pequeño. En el primer CIMA sobre los sistemas sanitarios, consta de cuatro temas y 12 horas de duración, donde se exponen las preguntas, los problemas a analizar, los contenidos conceptuales, procedimentales y actitudinales (figura 3).

Jornadas de Formación e Innovación Docente del Profesorado | № 2 (2019) Esta obra se distribuye con la licencia Creative Commons 
MARÍA DOLORES GUERRA-MARTÍN

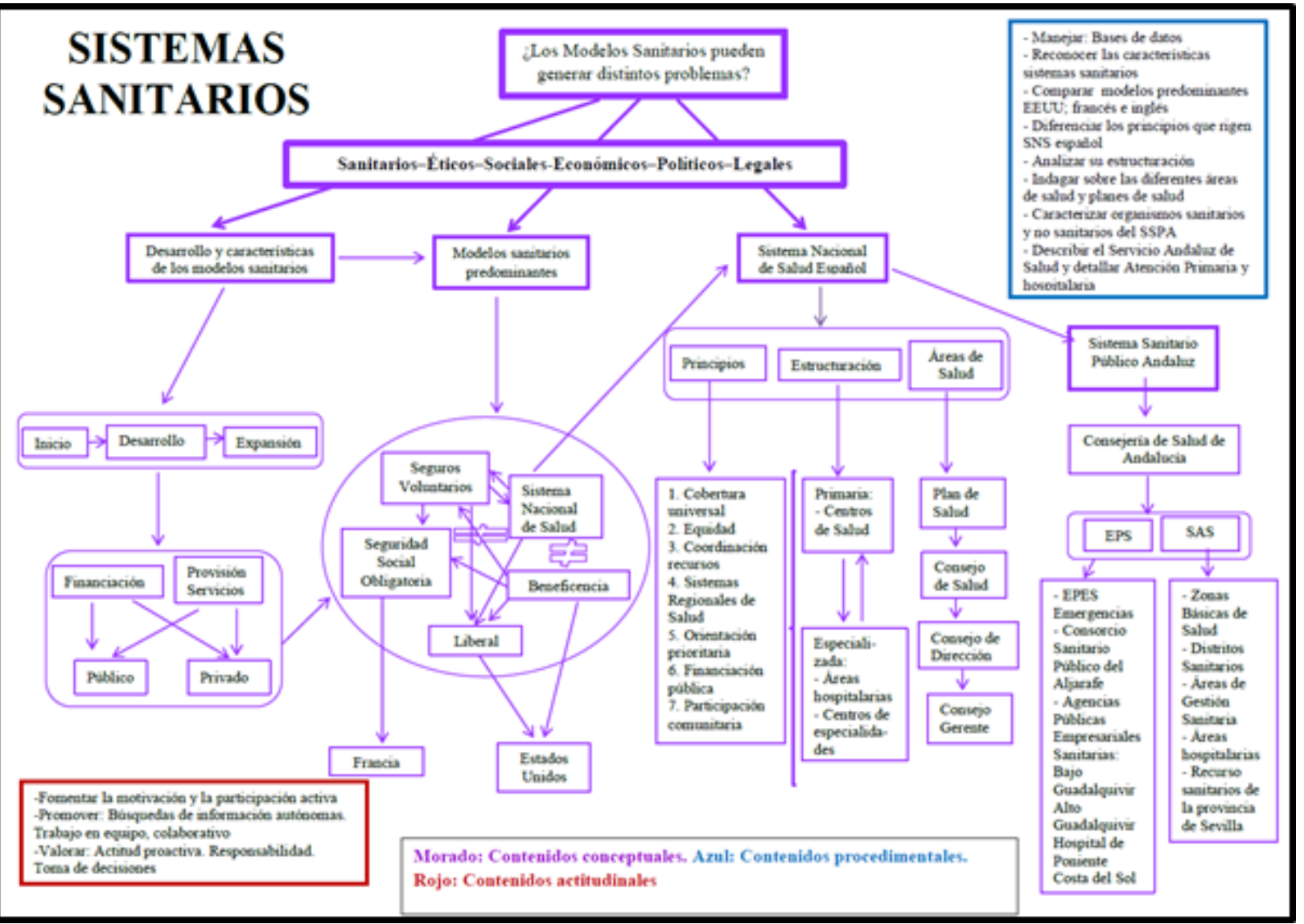

Figura 3: Mapa de contenidos conceptuales sobre los Sistemas Sanitarios (CIMA 1).

Jornadas de Formación e Innovación Docente del Profesorado I № 2 (2019)

c) (7) E $\Theta$ Esta obra se distribuye con la licencia Creative Commons 
El segundo CIMA sobre parte del Proceso Administrativo, consta también, de cuatro temas y 10 horas de duración, donde se exponen las preguntas, los contenidos conceptuales, procedimentales y actitudinales (figura 4).

\section{PROCESO ADMINISTRATIVO ¿Cuál es el método de resolución de problemas en la gestión sanitaria? ¿Qué etapas se siguen?}

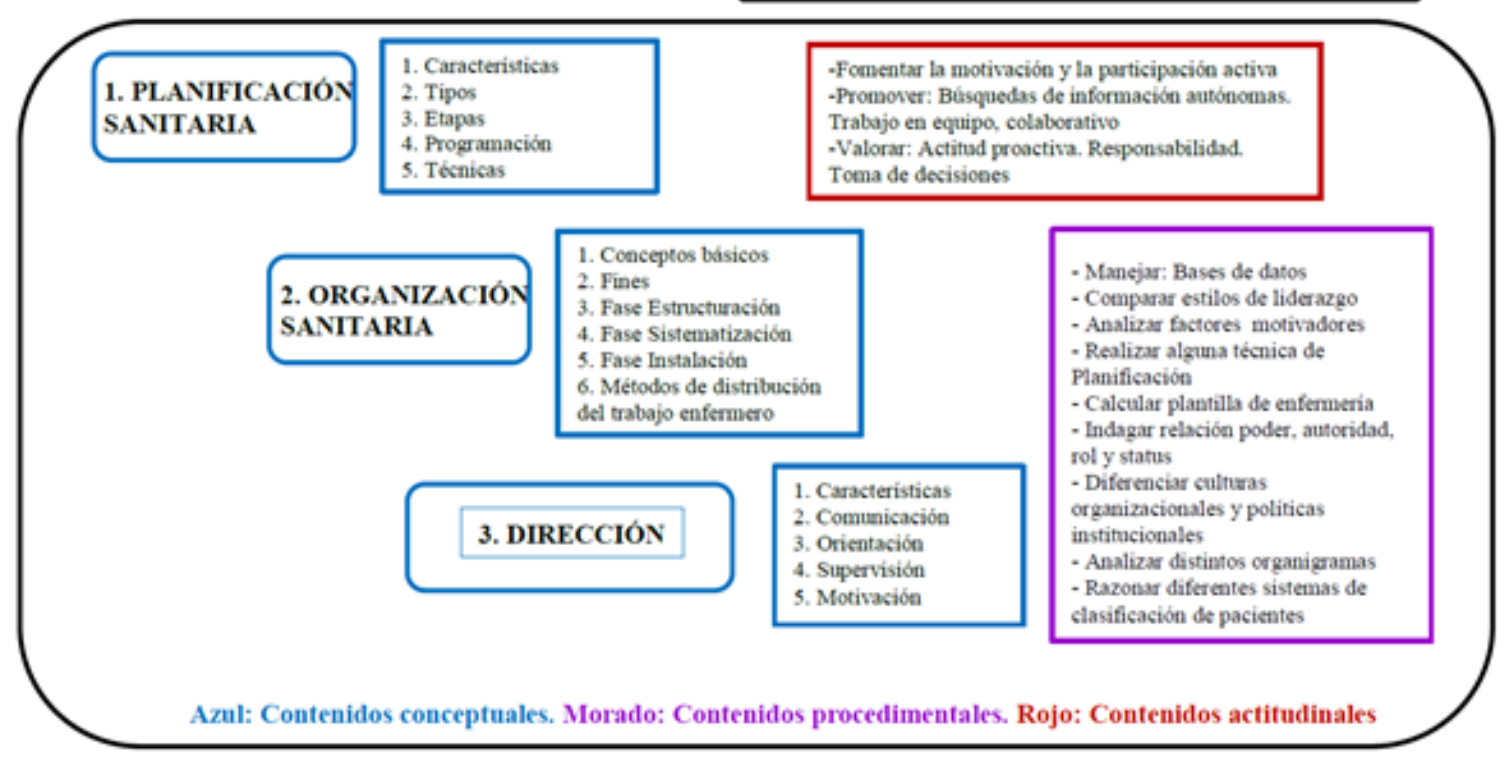

Figura 4: Mapa de contenidos conceptuales de parte del Proceso Administrativo (CIMA 2).

Jornadas de Formación e Innovación Docente del Profesorado I № 2 (2019) Esta obra se distribuye con la licencia Creative Commons Reconocimiento-NoComercial-SinObraDerivada Internacional (CC BY-NC-ND 4.0.) 
El tercer CIMA se ha llevado a cabo en seis talleres, de dos horas de duración cada uno, con un total de 12 horas, donde se exponen los contenidos conceptuales, procedimentales y actitudinales (Figura 5).

\section{TALLERES GESTIÓN SANITARIA}

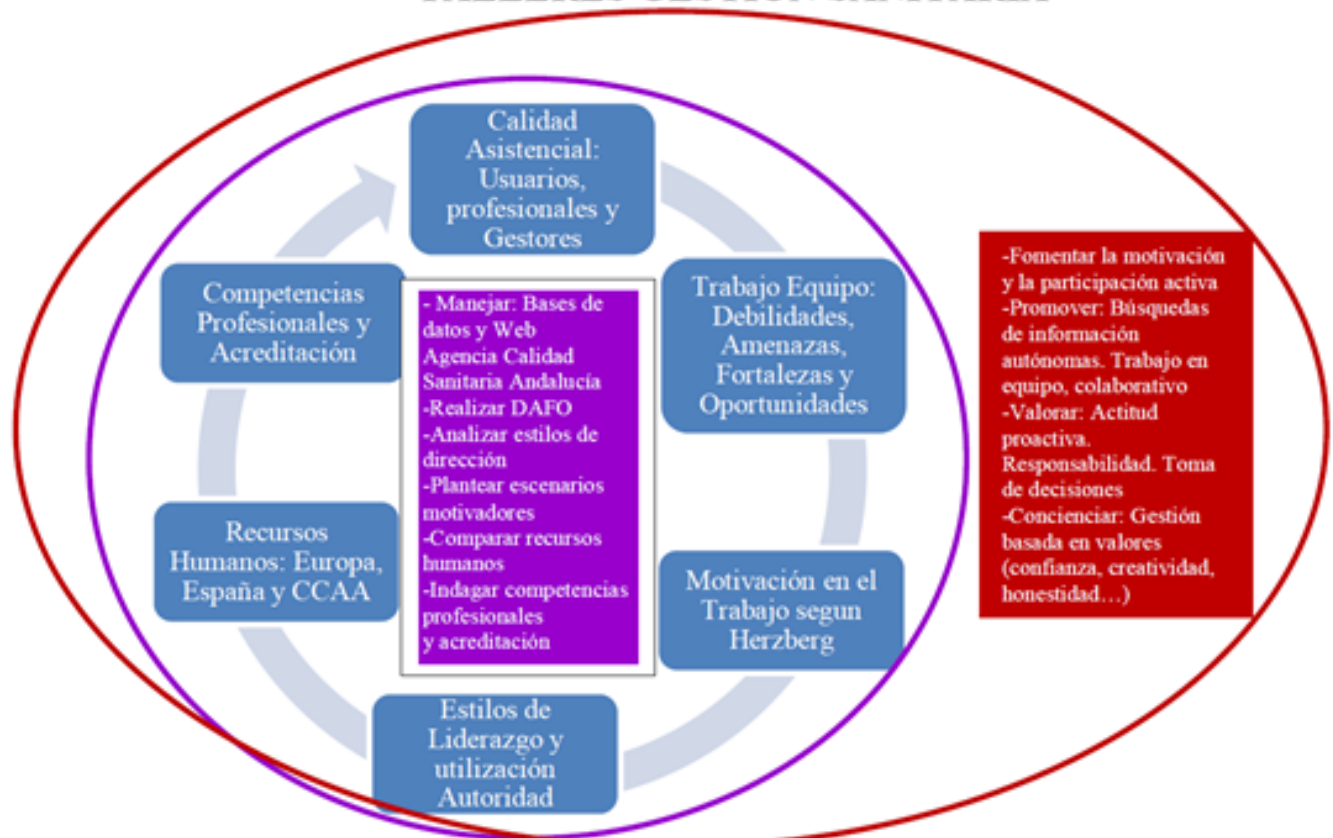

Azul: Contenidos conceptuales. Morado: Contenidos procedimentales. Rojo: Contenidos actitudinales

Figura 5: Mapa de contenidos conceptuales de los talleres en el grupo pequeño (CIMA 3).

Jornadas de Formación e Innovación Docente del Profesorado I № 2 (2019) Esta obra se distribuye con la licencia Creative Commons Reconocimiento-NoComercial-SinObraDerivada Internacional (CC BY-NC-ND 4.0.) 


\section{Secuencia de actividades}

En la tabla 1, se expone la temporalización de la secuencia de actividades, incluyendo las preguntas y los contenidos conceptuales del grupo grande (CIMA 1 y 2) y en la tabla 2, la del grupo pequeño (CIMA 3).

Tabla 1. Secuencia de actividades en grupo grande. CIMA 1 y 2.

\begin{tabular}{|c|c|c|c|}
\hline Fechas & Minutos & Preguntas & $\begin{array}{l}\text { Actividades y contenidos } \\
\text { conceptuales }\end{array}$ \\
\hline $26 / 09$ & 60 & \multicolumn{2}{|c|}{$\begin{array}{l}\text { Presentación asignatura y CIMAs } \\
\text { Cuestionarios previos de conocimientos Sistemas Sanitarios }\end{array}$} \\
\hline $27 / 09$ & 120 & \multirow{2}{*}{$\begin{array}{l}\text { ¿Qué características tienen } \\
\text { los Modelos Sanitarios: } \\
\text { Liberal, Seguros Voluntarios } \\
\text { y Seguros Sociales } \\
\text { Obligatorios? } \\
\text { ¿Qué características } \\
\text { tienen Modelos Sanitarios: } \\
\text { Asistencia Pública de } \\
\text { Beneficencia y Sistema } \\
\text { Nacional de Salud? }\end{array}$} & $\begin{array}{l}\text { Contenido conceptual: } \\
\text { Antecedentes de los } \\
\text { Sistemas Sanitarios. } \\
\text { Modelos Sanitarios }\end{array}$ \\
\hline $03 / 10$ & 60 & & $\begin{array}{l}\text { Contenido conceptual: } \\
\text { Sistemas de Salud de EEUU, } \\
\text { Francia y Reino Unido. }\end{array}$ \\
\hline $04 / 10$ & 120 & $\begin{array}{l}\text { ¿Cuáles son los principios } \\
\text { que rigen la Estructura y } \\
\text { Funcionamiento del Sistema } \\
\text { Nacional de Salud Español? }\end{array}$ & $\begin{array}{l}\text { Trabajo en equipo: } \\
\text { Elaboración preguntas temas } \\
1 \text { y } 2 \\
\text { Contenido conceptual: } \\
\text { Sistema Nacional de Salud } \\
\text { Español }\end{array}$ \\
\hline $10 / 10$ & 60 & $\begin{array}{l}\text { ¿Cuáles son los organismos } \\
\text { No sanitarios del SSPA, } \\
\text { adscritos a la Consejería de } \\
\text { Salud? }\end{array}$ & $\begin{array}{l}\text { Sistema Sanitario Público } \\
\text { de Andalucía: Organismos } \\
\text { No sanitarios adscritos a la } \\
\text { Consejería de Salud }\end{array}$ \\
\hline
\end{tabular}

Jornadas de Formación e Innovación Docente del Profesorado | № 2 (2019) Esta obra se distribuye con la licencia Creative Commons Reconocimiento-NoComercial-SinObraDerivada Internacional (CC BY-NC-ND 4.0.) 


\begin{tabular}{|c|c|c|c|}
\hline $11 / 10$ & 120 & $\begin{array}{l}\text { ¿Cuántas Agencias Públicas } \\
\text { Empresariales Sanitarias hay } \\
\text { en Andalucía y cuáles son } \\
\text { sus finalidades? }\end{array}$ & $\begin{array}{l}\text { Consorcio Sanitario Público } \\
\text { del Aljarafe. Empresa Pública } \\
\text { de Emergencias Sanitarias } \\
\text { y Agencias Públicas } \\
\text { Empresariales Sanitarias } \\
\text { Costa del Sol, Hospital } \\
\text { de Poniente, Hospital } \\
\text { Alto Guadalquivir y Bajo } \\
\text { Guadalquivir }\end{array}$ \\
\hline $17 / 10$ & 60 & $\begin{array}{l}\text { ¿Qué sabe sobre la } \\
\text { ordenación sanitaria del } \\
\text { Servicio Andaluz de Salud y } \\
\text { sobre los compromisos del } \\
\text { IV Plan Andaluz de Salud? }\end{array}$ & Servicio Andaluz de Salud. \\
\hline $18 / 10$ & 90 & $\begin{array}{l}\text { ¿Qué sabe sobre los Distritos } \\
\text { Sanitarios y sobre las Áreas } \\
\text { de Gestión Sanitaria? }\end{array}$ & $\begin{array}{l}\text { Atención Primaria (Centros } \\
\text { de Salud, Distritos } \\
\text { Sanitarios) Área de } \\
\text { Gestión Sanitaria, Atención } \\
\text { Hospitalaria. }\end{array}$ \\
\hline $18 / 10$ & 30 & \multicolumn{2}{|c|}{$\begin{array}{l}\text { Trabajo en equipo: Elaboración preguntas temas } 3 \text { y } 4 \\
\text { Realización de Kahoot de los temas del Sistemas Sanitario }\end{array}$} \\
\hline $24 / 10$ & 30 & \multicolumn{2}{|c|}{$\begin{array}{l}\text { Cuestionarios finales de conocimientos Sistemas Sanitarios } \\
\text { Cuestionarios previos de conocimientos del Proceso } \\
\text { Administrativo }\end{array}$} \\
\hline $24 / 10$ & 30 & $\begin{array}{l}\text { ¿Qué características tiene la } \\
\text { Planificación Sanitaria? }\end{array}$ & Planificación Sanitaria. \\
\hline $25 / 10$ & 120 & $\begin{array}{l}\text { ¿Cuáles son las Técnicas de } \\
\text { Planificación Sanitaria? }\end{array}$ & $\begin{array}{l}\text { Continuación Planificación } \\
\text { Sanitaria } \\
\text { Técnicas de Planificación. }\end{array}$ \\
\hline $31 / 0$ & 60 & $\begin{array}{l}\text { ¿Conoce los conceptos } \\
\text { básicos de la Organización } \\
\text { sanitaria? }\end{array}$ & $\begin{array}{l}\text { Organización Sanitaria: } \\
\text { Conceptos básicos. }\end{array}$ \\
\hline $07 / 11$ & 60 & $\begin{array}{l}\text { ¿Sabría describir las fases de } \\
\text { la Organización? } \\
\text { ¿Qué sabe de la estructura } \\
\text { de una organización? }\end{array}$ & $\begin{array}{l}\text { Fases de la organización y } \\
\text { tipos de Estructuras. }\end{array}$ \\
\hline $08 / 11$ & 90 & $\begin{array}{l}\text { ¿Conoce la organización de } \\
\text { los departamentos? } \\
\text { ¿Qué sabe de la } \\
\text { sistematización de una } \\
\text { organización? }\end{array}$ & $\begin{array}{l}\text { Organización de los } \\
\text { Departamentos y } \\
\text { Sistematización. }\end{array}$ \\
\hline
\end{tabular}




\begin{tabular}{|c|c|c|c|}
\hline $08 / 11$ & 30 & \multicolumn{2}{|c|}{ Trabajo en equipo: Elaboración preguntas temas 4 y 5} \\
\hline $14 / 11$ & 60 & $\begin{array}{l}\text { ¿Cuáles son los Sistemas } \\
\text { de Asignación de Cuidados } \\
\text { Enfermeros? }\end{array}$ & $\begin{array}{l}\text { Métodos de Distribución del } \\
\text { Trabajo Enfermero. }\end{array}$ \\
\hline $15 / 11$ & 70 & $\begin{array}{l}\text { ¿Qué elementos son } \\
\text { importantes dentro de } \\
\text { la etapa de la Dirección } \\
\text { Sanitaria? } \\
\text { ¿Cuáles son los } \\
\text { planteamientos de McGregor } \\
\text { en la motivación laboral? }\end{array}$ & $\begin{array}{l}\text { Elementos de la Dirección y } \\
\text { Motivación. }\end{array}$ \\
\hline $15 / 11$ & 30 & \multicolumn{2}{|c|}{$\begin{array}{l}\text { Trabajo en equipo: Elaboración preguntas temas } 6 \text { y } 7 \\
\text { Realización de Kahoot de los temas del Proceso } \\
\text { Administrativo }\end{array}$} \\
\hline $15 / 11$ & 20 & \multicolumn{2}{|c|}{$\begin{array}{l}\text { Cuestionarios finales de conocimientos del Proceso } \\
\text { Administrativo } \\
\text { Cuestionarios de opiniones y de satisfacción }\end{array}$} \\
\hline
\end{tabular}

Tabla 2. Secuencia de actividades en grupo pequeño (Talleres). CIMA 3.

\begin{tabular}{|c|c|l|l|}
\hline Fecha & Minutos & Preguntas & $\begin{array}{l}\text { Actividades y contenidos } \\
\text { conceptuales }\end{array}$ \\
\hline $30 / 09$ & 20 & $\begin{array}{l}\text { Presentación talleres de la parte de Gestión Sanitaria y CIMA } \\
\text { Cuestionarios previos de conocimientos }\end{array}$ \\
\hline 100 & $\begin{array}{l}\text { ¿Qué sabe sobre } \\
\text { los intereses de los } \\
\text { protagonistas del } \\
\text { sistema sanitario } \\
\text { en relación con los } \\
\text { elementos de la } \\
\text { calidad asistencial? }\end{array}$ & $\begin{array}{l}\text { Calidad asistencial, desde los } \\
\text { puntos de vista de los usuarios, } \\
\text { los profesionales sanitarios y los } \\
\text { gestores sanitarios. } \\
\text { Mediante juego de roles (rol de } \\
\text { usuario, rol profesional sanitario y } \\
\text { rol gestor sanitario). }\end{array}$ \\
\hline $07 / 10$ & 120 & $\begin{array}{l}\text { ¿Conoce las } \\
\text { debilidades, } \\
\text { amenazas, fortalezas } \\
\text { y oportunidades del } \\
\text { trabajo en equipo en } \\
\text { la práctica asistencial? }\end{array}$ & $\begin{array}{l}\text { Debilidades, amenazas, fortalezas } \\
\text { yoportunidades (DAFO) del trabajo } \\
\text { en equipo en la práctica asistencial. } \\
\text { Mediante búsquedas de } \\
\text { información (estudio, informe, } \\
\text { video, página web...). }\end{array}$ \\
\hline
\end{tabular}

Jornadas de Formación e Innovación Docente del Profesorado I № 2 (2019) Esta obra se distribuye con la licencia Creative Commons Reconocimiento-NoComercial-SinObraDerivada Internacional (CC BY-NC-ND 4.0.) 


\begin{tabular}{|c|c|c|c|}
\hline $14 / 10$ & & $\begin{array}{l}\text { ¿Qué conoce sobre los } \\
\text { factores que influyen } \\
\text { en la motivación } \\
\text { en el trabajo según } \\
\text { Herzberg? }\end{array}$ & $\begin{array}{l}\text { Factores que influyen en la } \\
\text { motivación en el trabajo según } \\
\text { Herzberg. } \\
\text { Mediante roll playing } \\
\text { (representación de situaciones que } \\
\text { motiven o desmotive en la práctica } \\
\text { asistencial). }\end{array}$ \\
\hline $21 / 10$ & & $\begin{array}{l}\text { ¿Qué conoce sobre } \\
\text { los diferentes estilos } \\
\text { de liderazgo según } \\
\text { la utilización de la } \\
\text { autoridad por el lider? }\end{array}$ & $\begin{array}{l}\text { Estilos de liderazgo según la } \\
\text { utilización de la autoridad del líder. } \\
\text { Mediante búsquedas de } \\
\text { información y análisis de los } \\
\text { distintos estilos encontrados en la } \\
\text { práctica asistencial }\end{array}$ \\
\hline $28 / 10$ & & $\begin{array}{l}\text { ¿Qué sabe sobre los } \\
\text { recursos humanos } \\
\text { sanitarios en España y } \\
\text { la Unión Europea? }\end{array}$ & $\begin{array}{l}\text { Recursos humanos sanitarios en } \\
\text { España y la Unión Europea. } \\
\text { Mediante el análisis de dos } \\
\text { documentos de trabajo, expuestos } \\
\text { en la enseñanza virtual. }\end{array}$ \\
\hline $04 / 11$ & 100 & $\begin{array}{l}\text { ¿Conoce la gestión } \\
\text { por competencias en } \\
\text { el ámbito sanitario } \\
\text { y la Agencia de } \\
\text { Calidad Sanitaria de } \\
\text { Andalucía? }\end{array}$ & $\begin{array}{l}\text { Gestión por competencias en el } \\
\text { ámbito sanitario y manejo de la } \\
\text { aplicación de la Agencia de Calidad } \\
\text { Sanitaria de Andalucía. } \\
\text { Mediante la lectura de un } \\
\text { documento de trabajo, y del } \\
\text { manejo de la web para localizar } \\
\text { evidencias y pruebas de buenas } \\
\text { prácticas de competencias } \\
\text { enfermeras. }\end{array}$ \\
\hline $4 / 11$ & 20 & \multicolumn{2}{|c|}{$\begin{array}{l}\text { Cuestionarios finales de conocimientos } \\
\text { Cuestionarios de opiniones y de satisfacción }\end{array}$} \\
\hline
\end{tabular}

\section{Cuestionario inicial-final}

Se han elaborado cuestionarios de conocimientos inicial y final en cada CIMA para conseguir un mayor acercamiento a los modelos metales de los estudiantes (Rivero y Porlán, 2017)). También, al finalizar los CIMA 1 y 2 de las clases teóricas, y el CIMA 3 de los talleres, se han realizado cuestionarios de opiniones y de satisfacción de los estudiantes. Además, durante todo el proceso de enseñanza-aprendizaje del CIMA 3 se ha llevado a cabo una 
evaluación continua, mediante la cual se han podido detectar errores y corregirlos sobre la marcha (feedfoward), para lo cual, la profesora ha contado con un cuaderno de campo, donde ha ido anotando la evolución y evaluación de los estudiantes, valorando tanto la participación (mediante observación) como los informes de equipo.

\section{Aplicación del CIMA}

\section{Relato resumido de sesiones}

Tanto las clases en grupo grande como los talleres en pequeño grupo, se desarrollaron tal y como se han expuesto en las tablas 1 y 2 de las actividades realizadas en los CIMA 1, 2 y 3. En la plataforma de la enseñanza virtual estaban colgados, desde el inicio del curso, todo el temario de la asignatura y también, otros materiales educativos. Para complementar esta información, decir que se les informaba a los estudiantes que debían traer leído el tema previamente a la asistencia al aula, o por lo menos, haber buscado alguna información o noticia relacionada con el mismo.

Al inicio de cada clase o taller se hacía una breve exposición de los contenidos que se iban a tratar en el aula y de las competencias a adquirir por los estudiantes. En grupo grande, cada dos temas se hacía un trabajo en equipo, donde estos buscaban información sobre los mismos y elaboraban preguntas con sus respuestas, por lo que desde el principio, se les informaba los días que tenían trabajo en equipo para que se trajesen sus portátiles o Tablet. Al finalizar el CIMA 1, se realizó un Kahoot con las preguntas que los estudiantes habían ido elaborando, lo mismo que se hizo al finalizar el CIMA 2. En los últimos minutos de las clases, hacía una síntesis y conclusiones del tema tratado, para finalizar recordando el tema de la siguiente clase. 
En grupo pequeño, los estudiantes trabajaron en equipo en los seis talleres, y se utilizaron distintas técnicas docentes, como juego de roles, teatralización, vídeos, manejo de páginas web, entre otras. En los talleres se recomendaba que los estudiantes trajesen sus portátiles o Tablet para las búsquedas de información, aunque existía la posibilidad de hacer dichas búsquedas en el aula de informática.

\section{Evaluación del aprendizaje de los estudiantes}

Para conocer los niveles de aprendizaje de los estudiantes, se elaboró una escalera de aprendizaje con cinco niveles, desde el nivel 1 para las respuestas de menor complejidad, hasta el nivel cinco para las que contenían una argumentación más elaborada, estos se exponen en la tabla 3.

Tabla 3. Niveles de la escalera de aprendizaje.

\begin{tabular}{|l|l|}
\hline Niveles & Respuestas según complejidad en la argumentación \\
\hline Nivel 1 & $\begin{array}{l}\text { No escribe nada o la contestación no es concordante con lo } \\
\text { consultado. }\end{array}$ \\
\hline Nivel 2 & $\begin{array}{l}\text { Escribe muy pocas ideas que apenas concuerdan con lo } \\
\text { consultado. }\end{array}$ \\
\hline Nivel 3 & $\begin{array}{l}\text { Escribe algunas ideas demostrando un conocimiento } \\
\text { suficiente sobre la materia. }\end{array}$ \\
\hline Nivel 4 & $\begin{array}{l}\text { Escribe bastantes ideas demostrando bastante conocimiento } \\
\text { sobre la materia. }\end{array}$ \\
\hline Nivel 5 & $\begin{array}{l}\text { Escribe muchas ideas demostrando mucho conocimiento } \\
\text { sobre la materia. }\end{array}$ \\
\hline
\end{tabular}

En todas las preguntas de los cuestionarios de conocimientos, las opciones de respuesta eran cinco desde 1 (nada) a 5 (mucho). En la tabla 4, se presentan las preguntas del CIMA 1 y los resultados (porcentajes y desviación típica), basados en la escalera de aprendizaje (5 niveles), 
tanto de los cuestionarios iniciales como de los finales. En la tabla 5 se presentan del CIMA 2 y en la tabla 6 del CIMA 3. Como se puede comprobar en las tres tablas, en todos los casos ha habido un aumento del conocimiento, bastante considerable. En cada tabla se ha puesto en negrita los resultados más elevados.

Tabla 4. Resultados de las preguntas iniciales y finales de los cuestionarios de conocimientos sobre el Sistema Sanitario (CIMA 1).

\begin{tabular}{|l|c|c|}
\hline Preguntas & Resultados Iniciales & Resultados Finales \\
\hline $\begin{array}{l}\text { ¿Qué características tienen los } \\
\text { Modelos Sanitarios: Liberal, Seguros } \\
\text { Voluntarios y Seguros Sociales } \\
\text { Obligatorios? }\end{array}$ & $1,08 \pm 0,269$ & $4,15 \pm 0,607$ \\
\hline $\begin{array}{l}\text { ¿Qué características tienen Modelos } \\
\text { Sanitarios: Asistencia Pública de } \\
\text { Beneficencia y Sistema Nacional de } \\
\text { Salud? }\end{array}$ & $1,06 \pm 0,235$ & $4,31 \pm 0,701$ \\
\hline $\begin{array}{l}\text { ¿Cuáles son los principios que rigen } \\
\text { la Estructura y Funcionamiento del } \\
\text { Sistema Nacional de Salud Español? }\end{array}$ & $1,02 \pm 0,139$ & $4,21 \pm 0,667$ \\
\hline $\begin{array}{l}\text { ¿Podría describir los organismos } \\
\text { no sanitarios del Sistema Sanitario }\end{array}$ & & $4,06 \pm 0,698$ \\
$\begin{array}{l}\text { Público de Andalucía y algunas de } \\
\text { las actividades que desarrolla cada } \\
\text { uno de ellos? }\end{array}$ & $1 \pm 0$ & $\mathbf{4 , 1 \pm 0 , 7 2 1}$ \\
\hline $\begin{array}{l}\text { ¿Cuántas Agencias Públicas } \\
\text { Empresariales Sanitarias hay en } \\
\text { Andalucía y finalidades de las } \\
\text { mismas? }\end{array}$ & $1,1 \pm 0,298$ & $4,65 \mathbf{3}$ \\
\hline $\begin{array}{l}\text { ¿Qué sabe sobre la ordenación } \\
\text { sanitaria de Andalucía? }\end{array}$ & $1 \pm 00,734$ \\
\hline $\begin{array}{l}\text { ¿Qué sabe de los compromisos del } \\
\text { IV Plan Andaluz de Salud? }\end{array}$ & & $4,17 \pm 0,585$ \\
\hline $\begin{array}{l}\text { ¿Qué sabe sobre los Distritos } \\
\text { Sanitarios? }\end{array}$ & & \\
\hline
\end{tabular}

Jornadas de Formación e Innovación Docente del Profesorado | № 2 (2019) Esta obra se distribuye con la licencia Creative Commons Reconocimiento-NoComercial-SinObraDerivada Internacional (CC BY-NC-ND 4.0.) 
Tabla 5. Resultados de las preguntas iniciales y finales de los cuestionarios de conocimientos sobre el Proceso Administrativo (CIMA 2).

\begin{tabular}{|c|c|c|}
\hline Preguntas & Resultados Iniciales & Resultados Finales \\
\hline $\begin{array}{l}\text { ¿Qué características tiene la } \\
\text { Planificación Sanitaria? }\end{array}$ & $1,32 \pm 0,61$ & $4,54 \pm 0,505$ \\
\hline $\begin{array}{l}\text { ¿Cuáles son las Técnicas de } \\
\text { Planificación Sanitaria? }\end{array}$ & $1,15 \pm 0,422$ & $3,98 \pm 0,57$ \\
\hline $\begin{array}{l}\text { ¿Podría describir los conceptos } \\
\text { básicos a tener en cuenta en la } \\
\text { Organización Sanitaria? }\end{array}$ & $1,2 \pm 0,459$ & $4,27 \pm 0,672$ \\
\hline $\begin{array}{l}\text { ¿Sabría describir las fases de la } \\
\text { organización? }\end{array}$ & $1,12 \pm 0,4$ & $4,17 \pm 0,704$ \\
\hline $\begin{array}{l}\text { ¿Qué sabe de la Estructura de una } \\
\text { Organización? }\end{array}$ & $1,12 \pm 0,4$ & $4,24 \pm 0,734$ \\
\hline $\begin{array}{l}\text { ¿Conoce la Organización de los } \\
\text { Departamentos? }\end{array}$ & $1,07 \pm 0,346$ & $4,17 \pm 0,704$ \\
\hline $\begin{array}{l}\text { ¿Qué sabe de la Sistematización de } \\
\text { una Organización? }\end{array}$ & $1,05 \pm 0,312$ & $4,17 \pm 0,704$ \\
\hline $\begin{array}{l}\text { ¿Cuáles son los Sistemas de } \\
\text { Asignación de Cuidados Enfermeros? }\end{array}$ & $1,22 \pm 0,419$ & $3,98 \pm 0,57$ \\
\hline $\begin{array}{l}\text { ¿Qué elementos son importantes a } \\
\text { tener en cuenta dentro de la etapa } \\
\text { de la Dirección Sanitaria? }\end{array}$ & $1,07 \pm 0,346$ & $3,98 \pm 0,57$ \\
\hline $\begin{array}{l}\text { ¿Cuáles son los planteamientos } \\
\text { de Mc Gregor en relación con la } \\
\text { Motivación Laboral? }\end{array}$ & $1,15 \pm 0,358$ & $3,98 \pm 0,57$ \\
\hline
\end{tabular}

Jornadas de Formación e Innovación Docente del Profesorado | № 2 (2019) Esta obra se distribuye con la licencia Creative Commons Reconocimiento-NoComercial-SinObraDerivada Internacional (CC BY-NC-ND 4.0.) 
Tabla 6. Resultados de las preguntas iniciales y finales de los cuestionarios de conocimientos sobre gestión sanitaria en la Talleres (CIMA 3).

\begin{tabular}{|c|c|c|}
\hline Preguntas & Resultados Iniciales & Resultados Finales \\
\hline $\begin{array}{l}\text { ¿Qué conocimientos tiene sobre } \\
\text { los intereses de los protagonistas } \\
\text { del sistema sanitario en relación } \\
\text { con los elementos de la calidad } \\
\text { asistencial? }\end{array}$ & $1 \pm 0$ & $4,08 \pm 0,494$ \\
\hline $\begin{array}{l}\text { ¿Qué conocimientos tiene sobre las } \\
\text { debilidades, amenazas, fortalezas } \\
\text { y oportunidades del trabajo en } \\
\text { equipo en la práctica asistencial? }\end{array}$ & $1,62 \pm 0,506$ & $4,15 \pm 0,555$ \\
\hline $\begin{array}{l}\text { ¿Qué conocimientos tiene sobre } \\
\text { los diferentes estilos de liderazgo } \\
\text { según la utilización de la autoridad } \\
\text { por el líder? }\end{array}$ & $1,08 \pm 0,277$ & $3,92 \pm 0,641$ \\
\hline $\begin{array}{l}\text { ¿Qué conocimientos tiene sobre } \\
\text { los factores que influyen en la } \\
\text { motivación en el trabajo según } \\
\text { Herzberg? }\end{array}$ & $1 \pm 0$ & $3,77 \pm 0,599$ \\
\hline $\begin{array}{l}\text { ¿Qué conocimientos tiene sobre } \\
\text { los recursos humanos sanitarios } \\
\text { de España y de la Unión Europea? }\end{array}$ & $1,08 \pm 0,277$ & $4,23 \pm 0,725$ \\
\hline $\begin{array}{l}\text { ¿Qué conocimientos tiene sobre } \\
\text { la gestión por competencias en el } \\
\text { ámbito sanitario? }\end{array}$ & $1 \pm 0$ & $3,85 \pm 0,689$ \\
\hline
\end{tabular}

En la tabla 7, se expone a modo de ejemplo, los niveles de desarrollo de conocimientos iniciales y finales, pormenorizado por cada uno de los estudiantes de los talleres (CIMA 3). Como se puede comprobar en dicha tabla, todos los estudiantes han tenido un avance en los conocimientos, destacando el estudiante 6 , que de seis preguntas, ha aumentado en tres de ellas, cuatro puntos en el nivel de conocimientos. 
Tabla 7. Niveles de desarrollo de conocimientos iniciales y finales CIMA 3.

\begin{tabular}{|c|c|c|c|c|c|c|c|c|c|c|c|c|c|c|c|c|c|c|}
\hline \multirow{3}{*}{ 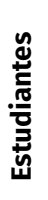 } & \multicolumn{18}{|c|}{ Preguntas } \\
\hline & \multicolumn{3}{|c|}{1} & \multicolumn{3}{|c|}{2} & \multicolumn{3}{|c|}{3} & \multicolumn{3}{|c|}{4} & \multicolumn{3}{|c|}{5} & \multicolumn{3}{|c|}{6} \\
\hline & $\mathbf{I}$ & $\mathbf{F}$ & $\mathbf{A}$ & I & $\mathbf{F}$ & $\mathbf{A}$ & I & $\mathbf{F}$ & A & $\mathbf{I}$ & $\mathbf{F}$ & A & I & $\mathbf{F}$ & A & $\mathbf{I}$ & $\mathbf{F}$ & A \\
\hline 1 & 1 & 4 & +3 & 2 & 5 & +3 & 1 & 4 & +3 & 1 & 4 & +3 & 1 & 4 & +3 & 1 & 4 & +3 \\
\hline 2 & 1 & 4 & +3 & 1 & 4 & +3 & 1 & 4 & +3 & 1 & 4 & +3 & 1 & 4 & +3 & 1 & 4 & +3 \\
\hline 3 & 1 & 4 & +3 & 1 & 5 & +4 & 1 & 4 & +3 & 1 & 4 & +3 & 1 & 4 & +3 & 1 & 5 & +4 \\
\hline 4 & 1 & 4 & +3 & 1 & 4 & +3 & 1 & 4 & +3 & 1 & 5 & +4 & 1 & 4 & +3 & 1 & 4 & +3 \\
\hline 5 & 1 & 5 & +4 & 1 & 4 & +3 & 1 & 5 & +4 & 1 & 4 & +3 & 1 & 4 & +3 & 1 & 4 & +3 \\
\hline 6 & 1 & 5 & +4 & 2 & 4 & +2 & 1 & 5 & +4 & 1 & 4 & +3 & 1 & 5 & +4 & 1 & 4 & +3 \\
\hline 7 & 1 & 3 & +2 & 2 & 4 & +2 & 1 & 3 & +2 & 1 & 4 & +3 & 2 & 3 & +3 & 1 & 3 & +2 \\
\hline 8 & 1 & 4 & +3 & 2 & 5 & +3 & 1 & 3 & +2 & 1 & 4 & +3 & 1 & 5 & +4 & 1 & 3 & +2 \\
\hline 9 & 1 & 4 & +3 & 2 & 4 & +2 & 1 & 4 & +3 & 1 & 3 & +2 & 1 & 3 & +2 & 1 & 5 & +4 \\
\hline 10 & 1 & 4 & +3 & 2 & 3 & +1 & 1 & 4 & +3 & 1 & 3 & +2 & 1 & 5 & +4 & 1 & 4 & +3 \\
\hline 11 & 1 & 4 & +3 & 1 & 4 & +3 & 1 & 4 & +3 & 1 & 4 & +3 & 1 & 5 & +4 & 1 & 3 & +2 \\
\hline 12 & 1 & 4 & +3 & 2 & 4 & +2 & 1 & 3 & +2 & 1 & 3 & +2 & 1 & 4 & +3 & 1 & 3 & +2 \\
\hline 13 & 1 & 4 & +3 & 2 & 4 & +2 & 1 & 4 & +3 & 1 & 3 & +2 & 1 & 5 & +4 & 1 & 4 & +3 \\
\hline
\end{tabular}

Con respecto a las preguntas del cuestionario de opiniones y satisfacción de los estudiantes, estos se han estructurado en cuatro bloques (trabajo del estudiantes, de los compañeros, del profesorado, de los talleres) y una pregunta sobre satisfacción. Este cuestionario se ha administrado tanto en la docencia en grupo grande como en pequeño grupo. Las opciones de respuesta han sido cinco desde 1 (nada) a 5 (mucho). Además, se preguntaron dos cuestiones abiertas, una sobre lo que más le ha gustado de las clases/talleres y otra sobre lo que mejorarían de las clases/talleres. En la tabla 7, se presentan las preguntas 
del CIMA 1 y 2 y los resultados (porcentajes y desviación típica) y en la tabla 8 el CIMA 3.

Como se puede comprobar en la tabla 7, los valores más elevados han estado relacionado con la adaptación a las necesidades del grupo y con la resolución de dudas, por parte de la profesora; y en la tabla 8, el más elevado ha sido la satisfacción con el trabajo de los compañeros.

Tabla 7. Resultados del cuestionario de opiniones y satisfacción con las clases (CIMA 1 y 2)

\begin{tabular}{|l|c|}
\hline Cuestiones & Resultados \\
\hline Opiniones sobre mi trabajo en los talleres & $3,68 \pm 0,582$ \\
\hline $\begin{array}{l}\text { He participado activamente en la consecución de los } \\
\text { objetivos }\end{array}$ & $3,58 \pm 0,507$ \\
\hline He aprendido sobre los temas & $3,32 \pm 0,582$ \\
\hline Me siento valorado por mis compañeros/as & $3,68 \pm 0,582$ \\
\hline Estoy satisfecho/a con mi trabajo & $3,37 \pm 0,761$ \\
\hline $\begin{array}{l}\text { Opiniones sobre el trabajo de mis compañeros/as en } \\
\text { los talleres }\end{array}$ & $3,16 \pm 0,602$ \\
\hline $\begin{array}{l}\text { Han participado activamente en la consecución de los } \\
\text { objetivos }\end{array}$ & $\mathbf{4 , 0 5} \pm 0,78$ \\
\hline $\begin{array}{l}\text { Estoy satisfecho/a con el trabajo de mis compañeros/ } \\
\text { as }\end{array}$ & $\mathbf{4 , 0 5} \pm 0,911$ \\
\hline Opiniones sobre la profesora de los talleres & $3,95 \pm 0,621$ \\
\hline $\begin{array}{l}\text { El profesorado se ha adaptado a las necesidades del } \\
\text { grupo }\end{array}$ & \begin{tabular}{l}
$\mathbf{4}$ \\
\hline $\begin{array}{l}\text { El profesorado me ha resuelto las dudas que tenía en } \\
\text { cada clase }\end{array}$
\end{tabular} \\
\hline $\begin{array}{l}\text { Me parece adecuada la actuación docente del } \\
\text { profesorado }\end{array}$ & \begin{tabular}{l} 
Opiniones sobre los talleres \\
\hline Me parece adecuada la metodología utilizada
\end{tabular} \\
\hline Me parecen adecuados los contenidos & 397 \\
\hline
\end{tabular}

Jornadas de Formación e Innovación Docente del Profesorado | № 2 (2019) Esta obra se distribuye con la licencia Creative Commons Reconocimiento-NoComercial-SinObraDerivada Internacional (CC BY-NC-ND 4.0.) 


\begin{tabular}{|l|c|}
\hline Me parece adecuada la evaluación & $3,79 \pm 0,787$ \\
\hline Satisfacción en general con los talleres & \\
\hline En general, me siento satisfecho/a con las clases & $3,95 \pm 0,78$ \\
\hline
\end{tabular}

Tabla 8. Resultados del cuestionario de opiniones y satisfacción con los talleres (CIMA 3)

\begin{tabular}{|c|c|}
\hline Cuestiones & Resultados \\
\hline \multicolumn{2}{|l|}{ Opiniones sobre mi trabajo en los talleres } \\
\hline $\begin{array}{l}\text { He participado activamente en la consecución de los } \\
\text { objetivos }\end{array}$ & $4,54 \pm 0,519$ \\
\hline He aprendido sobre los temas & $3,92 \pm 0,641$ \\
\hline Me siento valorado por mis compañeros/as & $4,31 \pm 0,751$ \\
\hline Estoy satisfecho/a con mi trabajo & $4,31 \pm 0,751$ \\
\hline \multicolumn{2}{|l|}{$\begin{array}{l}\text { Opiniones sobre el trabajo de mis compañeros/as en los } \\
\text { talleres }\end{array}$} \\
\hline $\begin{array}{l}\text { Han participado activamente en la consecución de los } \\
\text { objetivos }\end{array}$ & $4,54 \pm 0,519$ \\
\hline Estoy satisfecho/a con el trabajo de mis compañeros/as & $4,62 \pm 0,506$ \\
\hline \multicolumn{2}{|l|}{ Opiniones sobre el profesorado de los talleres } \\
\hline $\begin{array}{l}\text { El profesorado se ha adaptado a las necesidades del } \\
\text { grupo }\end{array}$ & $4,31 \pm 0,48$ \\
\hline $\begin{array}{l}\text { El profesorado me ha resuelto las dudas que tenía en } \\
\text { cada clase }\end{array}$ & $4,46 \pm 0,519$ \\
\hline $\begin{array}{l}\text { Me parece adecuada la actuación docente del } \\
\text { profesorado }\end{array}$ & $4,23 \pm 0,599$ \\
\hline \multicolumn{2}{|l|}{ Opiniones sobre los talleres } \\
\hline Me parece adecuada la metodología utilizada & $4,31 \pm 0,63$ \\
\hline Me parecen adecuados los contenidos & $4,08 \pm 0,862$ \\
\hline Me parece adecuada la evaluación & $4 \pm 0,913$ \\
\hline \multicolumn{2}{|l|}{ Satisfacción en general con los talleres } \\
\hline En general, me siento satisfecho/a con las clases & $4 \pm 0,707$ \\
\hline
\end{tabular}

Jornadas de Formación e Innovación Docente del Profesorado | № 2 (2019) Esta obra se distribuye con la licencia Creative Commons Reconocimiento-NoComercial-SinObraDerivada Internacional (CC BY-NC-ND 4.0.) 
Por último, a continuación se presenta una sintesis de los comentarios que han expresado los estudiantes, en las preguntas abiertas del cuestionario de opiniones y satisfacción, tanto de las clases (CIMA 1 y 2), como de los talleres (CIMA 3).

\section{Preguntas abiertas del cuestionario de opiniones de los estudiantes sobre las clases (CIMA 1 y 2).}

Algunos de los comentarios sobre lo que más le ha gustado de las clases, han sido, la dinámica de las clases, hacer Kahoot con el modelo de preguntas del examen, las preguntas tipo test sobre los temas que te obliga a leértelos y aprender y se te quedan muchas partes de los temas con mayor facilidad, el hacer las clases participativas y reforzar los contenidos anteriores para afianzarlos al acabar los bloques, la profesora está muy involucrada en responder las dudas de los alumnos y en saber si han entendido el temario, así como los recursos y medios que nos ha facilitado para aprender, además, de los ejemplos y las explicaciones que nos pone, que se resuelvan todas las dudas que van surgiendo siempre y que se explique todo las veces que haga falta hasta que se entienda, el aprendizaje activo, la resolución de las dudas y repasar los conceptos importantes de cada tema.

En cuanto a lo que mejorarian de las clases: ver algún vídeo en clase, o algo similar, por ejemplo, que nos den alguna charla, intentar fomentar más la asistencia (tenemos mucho trabajo de las asignaturas, de los talleres, unido a las prácticas, al final no nos queda tiempo para estudiar), que los estudiantes nos leamos los temas antes de cada clase, los documentos a estudiar son largos, por lo que, sintetizar la teoría sería una opción muy válida para que la asignatura fuese más llevadera, y que la asignatura en sí no llama la atención.

Jornadas de Formación e Innovación Docente del Profesorado | № 2 (2019) Esta obra se distribuye con la licencia Creative Commons Reconocimiento-NoComercial-SinObraDerivada 


\section{Preguntas abiertas del cuestionario de opiniones de los estudiantes sobre los talleres (CIMA 3).}

Algunos de los comentarios sobre lo que más le ha gustado de los talleres, han sido las dinámicas de los talleres, el trabajo en equipo, que han tenido tiempo para preparar las tareas de cada taller, la motivación por parte de la profesora, la buena actitud de los compañeros y la diversidad de los talleres (juego de roles, teatralización, videos, entre otros) y el aprendizaje "casi jugando".

En cuanto a lo que mejorarían de los talleres, refieren que se trabajen aspectos del temario de cara al examen, casos prácticos de cara al futuro laboral, que no haya que entregar informes tras cada taller, que haya menos contenido teórico. Además, han surgido dos quejas de los estudiantes, relacionadas con la estructura, una relacionada con el horario de tarde (que ya están cansados) y otra con la falta de enchufes en el espacio asignado para la docencia.

\section{Evaluación de los CIMA 1, 2 y 3}

A lo largo del curso se ha ido haciendo una evaluación con fines formativos orientada a la mejora del aprendizaje de los estudiantes, donde estos han participado de forma activa y donde se ha creado un ambiente de trabajo basado en el diálogo, respecto y confianza para que los estudiantes se expresen con libertad, y donde el error no se ha visto como un fallo en el proceso de enseñanza, sino más bien como una posibilidad de generar nuevos conocimientos o de afianzarlos. Al utilizar los cuestionarios de conocimientos con preguntas abiertas, basado en los aspectos más relevantes, como docente he tenido la posibilidad de conocer algo más los procesos mentales de los estudiantes. Toda esta información la he analizado con rigor, basándome en los niveles de la escalera de aprendizaje 
expuesta anteriormente, la cual está ordenada según la complejidad de las respuestas proporcionadas por los estudiantes (Rivero y Porlán, 2017). En este sentido, se ha observado un aumento de los conocimientos en todas las preguntas de cada uno de los CIMA, teniendo en cuenta que estos partían mayoritariamente, de un nivel bajo (niveles 1 y 2 de la escalera de aprendizaje). Con respecto a los resultados de los cuestionarios de opiniones y satisfacción, referir que las puntuaciones han sido algo más elevadas en grupo pequeño que en grupo grande.

A modo de conclusión, decir que los estudiantes han sabido desde el inicio de las clases, cómo se les va a evaluar en la asignatura, lo cual ha guiado su proceso de enseñanza-aprendizaje. Estos han manifestado una gran satisfacción, resaltando la alta valoración que le han dado al trabajo de los compañeros en el grupo pequeño (CIMA 3). Además, ha habido una alta participación de los estudiantes, los cuales, han tenido una actitud proactiva, en mayor medida en los talleres, al ser el grupo menor, lo que les ha hecho sentirse cómodos a la hora de expresar sus dudas, preguntas e inquietudes.

Jornadas de Formación e Innovación Docente del Profesorado I № 2 (2019) Esta obra se distribuye con la licencia Creative Commons 


\section{Referencias bibliográficas}

De Alba, N. y Porlán, R. (2017). La metodología de enseñanza. En R. Porlán (Coord.), Enseñanza universitaria. Cómo mejorarla (37-23). Madrid: Ediciones Morata.

Gil-Galván, R. (2018). El uso del aprendizaje basado en problemas en la enseñanza universitaria. Análisis de las competencias adquiridas y su impacto. Revista mexicana de investigación educativa, 23 (76): 73-93. Recuperado de http://www.scielo.org.mx/scielo.php?script=sci arttext\&pid=S1405-66662018000100073\&ln$g=e s \& t \operatorname{lng}=e s$

Guerra, M. D. (2009). Opiniones de los Estudiantes de Enfermería sobre el Aprendizaje Basado en Problemas. Enfermería Global 8 (3): 1-18.

Guerra-Martín, M. D., Fernández-Rodríguez, V., Lima-Serrano, M. y Rodríguez-Borrego, M. A. (2012). Proyecto de tutorización de la docencia (feedforward) en grupos pequeños para mejorar el proceso de aprendizaje. Actas Congreso Internacional de Docencia universitaria e Innovación (CIDUI). Recuperado de http://www. cidui.org/revista-cidui12/index.php/cidui12/article/ view/469/472

Fernández-Gámez, D. y Guerra-Martín, M. D. (2016). Aprendizaje inverso en formación profesional: opiniones de los estudiantes. International Journal of Technology and Educational Innovation, Innoeduca 2 (1): 29-37. Recuperado de http://www.revistas.uma.es/index.php/ innoeduca/article/view/1048/1888

Guerra-Martín, M. D. (2015). Ciclo de mejora en la asignatura Ética y Gestión de Enfermería, con énfasis en la construcción de una rúbrica de evaluación, En R. Porlán y E. Navarro (Coords.). Libro de Actas de las II Jornadas de Docencia Universitaria (3-14). Sevilla: Instituto de Ciencias de la Educación. Recuperado de https: / / www.researchgate.net/publication/296706973 Ciclo de mejora en la asignatura Etica y Gestion de enfermeria con enfasis en la construccion de una rubrica de evaluacion

Guerra-Martín, M. D. (2017). Desarrollo y evaluación de un ciclo de mejora aplicado en los seminarios de la asignatura de Ética y Gestión de Enfermería. En R. Porlán y E. Navarro (Coords.), Libro de Actas de las IV Jornadas de Formación e Innovación Docente (1.105-1.115). Sevilla: Instituto de Ciencias de la Educación. Recuperado de https://www.researchgate.net/publication/337481995 DESARROLLO Y EVALUACION DE UN CICLO DE ME-

Jornadas de Formación e Innovación Docente del Profesorado | № 2 (2019) Esta obra se distribuye con la licencia Creative Commons Reconocimiento-NoComercial-SinObraDerivada Internacional (CC BY-NC-ND 4.0.) 
IORA APLICADO EN LOS SEMINARIOS DE LA ASIGNATURA DE ETICA Y GESTION DE ENFERMERIA

Guerra-Martín, M. D. (2018). Ciclo de Mejora en la docencia de Gestión de Enfermería, mediante el trabajo en equipo y colaborativo. En R. Porlán y E. Navarro (Coords.), Jornadas de Formación e Innovación Docente del profesorado (1.657-1.675). Sevilla: Instituto de Ciencias de la Educación. Recuperado de

https://www.researchgate.net/publication/331577527 Ciclo de mejora en la docencia de gestion de enfermeria mediante el trabajo en equipo y colaborativo

Padilla-Carmona, M. T., Gil-Flores, J., Rodríguez-Santero, J., Torres-Gordillo, J. J., Clares-Lopez, J. (2010). Evaluando el sistema de evaluación del aprendizaje universitario: análisis documental aplicado al caso de la Universidad de Sevilla. Revista Iberoamericana de Educación, $53(3): 1-14$.

Porlán, R. (Coord), Vázquez, J., Solís, E., Martín, R., Pineda, J. A., Duarte, O., De Alba, N., García, E., Navarro, E., Rivero, A., García, F. F., Feria, B., Guerra-Martín, M. D., Fuentes, A., Mora, J. P. y Herrera, J. A. (2017). Enseñanza universitaria. Cómo mejorarla. Ediciones Morata. Sevilla.

Rivero, A. y Porlán, R. (2017). La evaluación en la enseñanza universitaria. En R. Porlán (Coord.), Enseñanza universitaria. Cómo mejorarla (73-91). Madrid: Ediciones Morata. Tourón, J. y Santiago, R. (2015). El modelo Flipped Learning y el desarrollo del talento en la escuela. Revista de Educación 368: 196-231.

Jornadas de Formación e Innovación Docente del Profesorado | № 2 (2019) Esta obra se distribuye con la licencia Creative Commons Reconocimiento-NoComercial-SinObraDerivada Internacional (CC BY-NC-ND 4.0.) 